\title{
Evidence that the bifunctional redox factor / AP endonuclease Ref-1 is an anti-apoptotic protein associated with differentiation in the developing retina
}

\author{
LB Chiarini ${ }^{\star, 1}$, FG Freitas ${ }^{1}$, H Petrs-Silva ${ }^{1}$ and R Linden ${ }^{1}$ \\ 1 Instituto de Biofisica da UFRJ, Rio de Janeiro, Brasil \\ * Corresponding author: LB Chiarini, Instituto de Biofisica da UFRJ, CCS, bloco \\ G, Cidade Universitária, 21949-900, Rio de Janeiro, Brasil. \\ E-mail: chiarini@biof.ufrj.br
}

Received 15.1.99; revised 8.9.99; accepted 16.11 .99 Edited by $\mathrm{J}$ Reed

\begin{abstract}
Retinal cell differentiation leads to resistance to apoptosis induced by inhibition of protein synthesis, suggesting the accumulation of anti-apoptotic proteins. The redox factor/AP endonuclease Ref-1 (APE, APEX, HAP1) affects both DNA repair and the activity of various transcription factors, and controls sensitivity to genotoxic insults. We studied the expression of Ref-1 in the retina and brain of developing rats. Ref-1 immunoreactivity increased progressively within the nucleus of differentiating retinal cells, whereas it decreased in the developing hippocampal formation. During both natural and experimentally-induced cell death, Ref-1 disappeared from the nucleus of apoptotic cells. Degradation of Ref-1 in axotomized ganglion cells preceded the morphological characteristics of apoptosis. The sensitivity to apoptosis triggered by either thapsigargin or okadaic acid was the highest in photoreceptors, that contain the least Ref-1 among differentiated retinal cells. In both these differentiated cell types, inhibition of protein synthesis prevented the loss of Ref1 and rescued the neurons. The data suggest that Ref- 1 is an anti-apoptotic protein associated with cell differentiation in the retina. Cell Death and Differentiation (2000) 7, 272-281.
\end{abstract}

Keywords: programmed cell death; apoptosis; neurogenesis; central nervous system; DNA repair

Abbreviations: AP, apurinic/apyrimidinic; APE, AP endonuclease; HAP1, human AP1; NFG, nerve growth factor; Ref-1, redox factor 1

\section{Introduction}

Neural development proceeds through successive stages of proliferation, differentiation and programmed cell death. ${ }^{1}$ Coordination of these events involves changing patterns of gene expression, the mechanisms of which are still poorly understood. We use the developing retina as a model tissue to study patterns of gene expression associated with major events of development in the central nervous system, such as neuronal differentiation and programmed cell death.
The retina contains multiple, interactive cell types organized in well defined layers, which constitute basic features of several areas of the central nervous system. Retinal tissue is amenable to in vitro manipulation, for example in the form of histotypical explants that have been used to study mechanisms of programmed cell death while preserving the structure, connectivity and extracellular matrix similar to the retina in situ. ${ }^{2,3}$

The retina of newborn rats contains a layer of postmitotic ganglion cells, separated by an inner plexiform layer of processes from the early differentiating amacrine cells. The amacrine cells are continuous with the neuroblastic layer. The latter contains both postmitotic undifferentiated cells and proliferating neuroblasts and glioblasts, as well as early differentiating horizontal cells and a few cones. ${ }^{2}$ In the mature retina the ganglion cell layer contains both ganglion cells and displaced amacrines, the inner nuclear layer contains amacrine, bipolar and horizontal cells and Muller glia, and the outer nuclear layer houses the cell bodies of the photoreceptors (rods and cones). The cell body layers are now separated by an inner and an outer plexiform layers, which contain cell processes and synapses. ${ }^{4}$

Following the last round of cell division, post-mitotic neurons migrate to appropriate locations, differentiate and connect with both target and afferent neurons. Young neurons compete for trophic support, and those that fail to relate to appropriate partners undergo programmed cell death. ${ }^{5}$ In the retina, naturally occurring cell death in the form of apoptosis is detected initially in the ganglion cell layer and proceeds towards the outer nuclear layer, thus following the gradient of differentiation across the depth of the developing retinal tissue. ${ }^{6}$

The transition from the cell cycle to the fully differentiated state appears to be a critical period for neuronal survival. For example, it has been shown that post-mitotic cells shortly after their last round of cell division are particularly sensitive to apoptosis induced by inhibition of protein synthesis in the developing retina in vitro. ${ }^{2}$ During this period major changes in the repertoire of gene expression take place leading retinal cells both to the expression of specific phenotypic markers, ${ }^{7}$ and to a strict dependence on neurotrophic factors. ${ }^{8}$ These changing patterns occur under the control of several transcription factors, the activity of which is, in turn, modulated by mechanisms such as phosphorylation and redox reactions.

The protein Ref- 1 , originally reported as the apurinic/ apyrimidinic repair endonuclease APE/APEX/HAP- $1,{ }^{9,10}$ is also a redox factor. ${ }^{11}$ The two functions are independently performed by Ref-1, in which the carboxy terminal domain has endonuclease activity while the amino terminal domain has redox activity. ${ }^{12}$ Homozygous deletion of the ref-1 gene is lethal at embryonic day 6.5 in the mouse, ${ }^{13}$ thus underscoring its importance for embryonic development. 
Ref-1 is a widespread regulator of the activity of transcription factors, such as Jun and Fos families, NF- $\kappa$ B and $p 53,{ }^{11,14,15}$ which control multiple events in the life cycle of various cell types, ${ }^{16,17}$ inclusive of apoptosis. Down-regulation of Ref-1 by transfection of anti-sense cDNA into either glioma or HeLa cells was followed by increased sensitivity to genotoxic insults such as oxidative stress, as shown by decreased clonogenicity. ${ }^{18,19}$ Nonetheless, both increased as well as decreased levels of Ref1 were shown associated with apoptosis. ${ }^{20-22}$

The expression of both Ref- 1 mRNA and protein have been reported to be heterogeneous among various cell populations of the central nervous system. ${ }^{23,24}$ Due to the evidence for its critical role in developmental mechanisms and to its capacity to modulate the activity of transcription factors involved in the cell cycle, differentiation and cell death pathways, we studied the association of Ref-1 with differentiation and apoptosis in the developing retina.

\section{Results}

\section{Ref-1 accompanied differentiation of the retinal cells}

We analyzed the expression of Ref- 1 by immunohistochemistry in sections of the retina from rats at birth (postnatal day 0-P0), P6-P7, P13, P27 and P45.

Ref- 1 immunoreactivity in the retina of newborn rats $(P 0)$ was found predominantly in the ganglion cell layer, and in the early differentiating amacrine cells adjacent to the inner plexiform layer (Figure 1A). These cells also stain for calretinin in the newborn rat retina. The neuroblastic layer was faintly labeled with the antibody. A few evenly spaced large cells located at about the middle of the neuroblastic layer in a row aligned in parallel with the retinal pigment epithelium (RPE) were heavily stained for Ref-1. Their size, position and
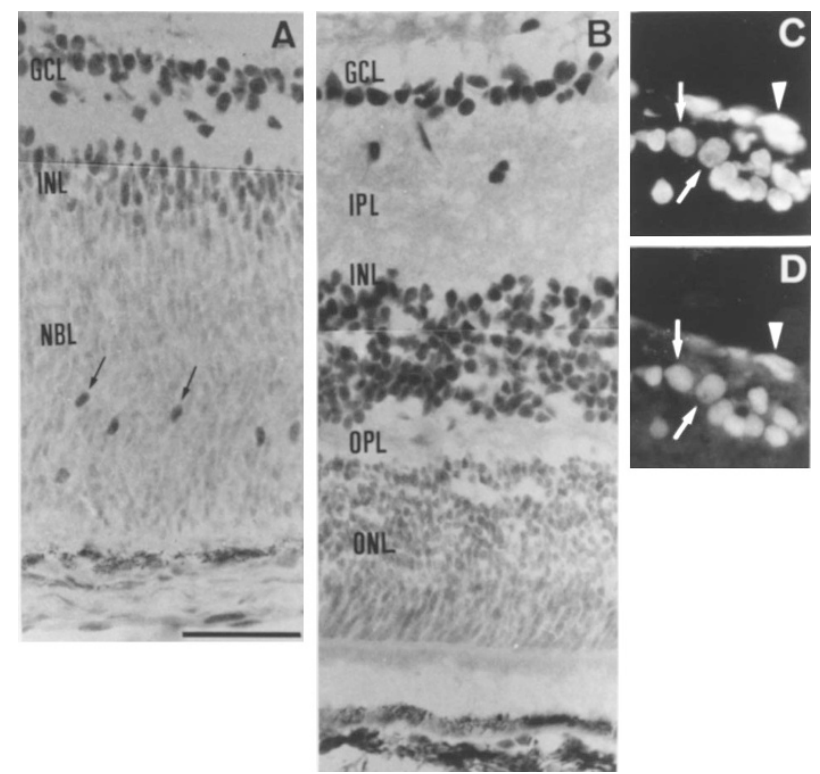

Figure 1 Transverse sections from the rat retina $(\mathbf{A}, \mathbf{B})$, stained with an antibody to Ref-1. (A) Newborn rat (P0). Notice the labeling in the ganglion cell layer (GCL), amacrine cells in the inner nuclear layer (INL) and horizontal cells (arrows). Calibration bar $=30 \mu \mathrm{m}$. (B) P13 retina. Notice the increased labeling of the ganglion cell and inner nuclear layers, and less intense labeling of the outer nuclear layer (ONL). Abbreviations: NBL=neuroblastic layer; IPL=inner plexiform layer; OPL=outer plexiform layer. RPE=retinal pigment epithelium (the dark staining is due to melanin). (C, D) Bisbenzimide staining of DNA (C) and overlapping staining for Ref- 1 (D) in the ganglion cell layer of a newborn rat retina. Notice that Ref- 1 staining is nuclear both in the ganglion cells (arrows) and in vascular endothelium (arrowhead)
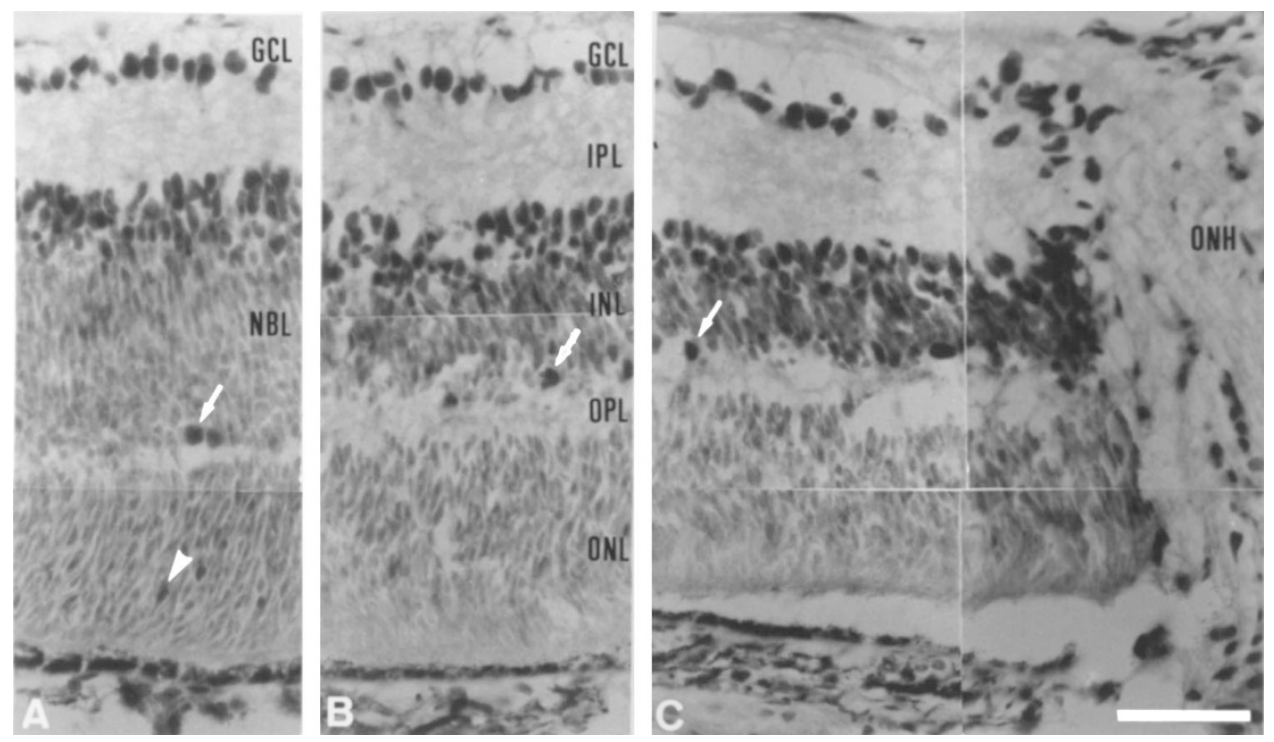

Figure 2 Immunohistochemistry for Ref-1 in a P7 retina. The photomicrographs were taken from peripheral $(\mathbf{A})$, intermediate $(\mathbf{B})$ and central $(\mathbf{C})$ portions of the retina. Notice the gradient of Ref-1 staining, coinciding with the most differentiated cells in each portion of the retina, particularly within the stratum containing the inner nuclear and neuroblastic layers. Arrows point to horizontal cells, and arrowhead indicates an elongated cell strongly stained for Ref-1 within the ONL (see text). Abbreviations as in Figure 1, plus $\mathrm{ONH}=$ optic nerve head. Calibration bar $=30 \mu \mathrm{m}$ 
alignment indicate that they are horizontal cells. Several elongated cells were also intensely stained for Ref- 1 close to the outer margin of the retina. These cells did not stain with an antibody for rhodopsin that, nonetheless, labeled a few adjacent rods at this age. Both the horizontal cells and the Ref- 1 positive external cells co-localized Ref- 1 and calbindin. Thus the external cells may be either migrating horizontal cells on their way to the outer rim of the inner nuclear layer, or cones transiently expressing calbindin. The overall pattern of immunoreactivity to Ref-1 corresponds to the most differentiated cells in the newborn rat retina.

In the retina of 2 week-old or older rats, the intensity of the immunoreactivity generally increased (Figure 1B). Labeling was the most intense in the ganglion cell and inner nuclear layers, with relatively less staining in the outer nuclear layer. Staining of the photoreceptors was, nonetheless, more intense than in the neuroblastic layer of younger rats. In tissue from both P27 and P45 rats the pattern of immunoreactivity for Ref-1 was similar to that at P13 (not shown).

Neither the inner nor the outer plexiform layers were stained. Simultaneous staining with the DNA labeling dye bisbenzimide, and immunohistochemistry showed that Ref1 was concentrated in the nucleus of the retinal cells (Figure 1C,D).

At about 1 week after birth, the neuroblastic layer containing proliferating cells is restricted to peripheral portions of the retina, and occupies an intermediate strip, between the inner nuclear layer (INL) and the emerging outer plexiform layer (OPL). In sections of the retina from $\mathrm{P} 7$ rats, immunoreactivity for Ref-1 appeared in the ganglion cell, inner and outer nuclear layers. The immunoreactivity was less in the INL than in the GCL, except for the heavily labeled horizontal cells. Both the emerging outer nuclear layer and the neuroblastic layer were less intensely stained than the remaining retinal layers.

At this age, the central portions of the retina, close to the optic disk, are well advanced in the course of differentiation compared with the retinal periphery, as shown by the progressively more extensive incorporation of BrdU towards the periphery. The centro-peripheral gradient of retinal maturation is reflected in the distribution of Ref-1 immunoreactivity. In the least differentiated far periphery of the retina, staining for Ref-1 was the least intense, compared with the more advanced central parts (Figure 2 and 3). The pattern of staining in the periphery was similar to that of newborn rats, mainly in ganglion cells, the few rows of differentiated amacrine cells, and the horizontal cells. In turn, staining in the central retinal was heavy across the whole inner nuclear layer. Thus, the centroperipheral gradient of Ref-1 staining reproduced the sequence of maturation observed with increasing age.

In Western blots we found an increase in the retinal content of Ref-1 during postnatal development (Figure 4). A single band of approximately $37 \mathrm{KDa}$ was detected with the Ref-1 antibody, which corresponds to the reported molecular weight of Ref- $1 .{ }^{11}$ In four out of five experiments with proteins extracted from the retinae of rats at P0/P6, P0/P13 and P0/P22, densitometric readings of the Ref-1

\section{Erk2}

\section{Ref-1}
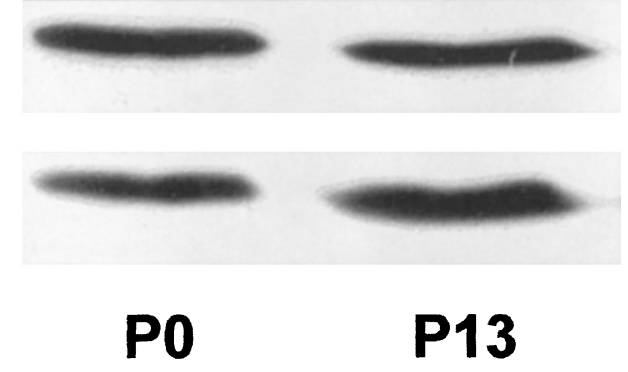

Figure 4 Western blot for Ref-1. Samples of $40 \mu \mathrm{g}$ each from the retina at either $\mathrm{P} 0$ or $\mathrm{P} 13$ were run in $12.5 \%$ polyacrylamide gels and blotted with the same antibody used for immunohistochemistry, then stripped and reblotted with an antibody to Erk2
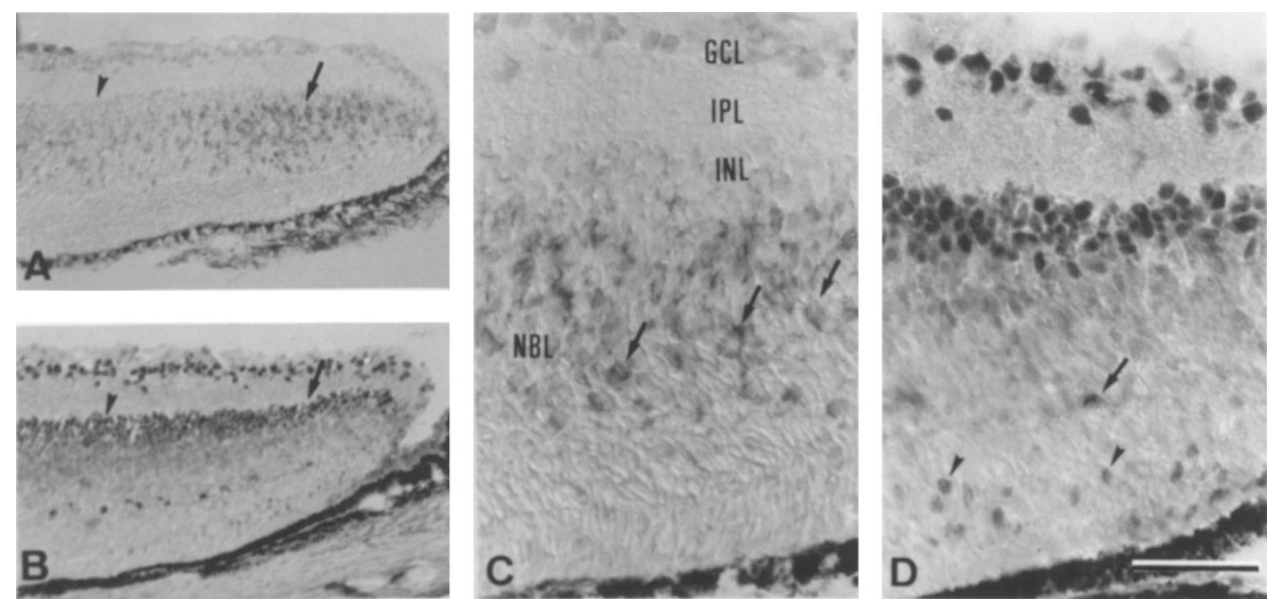

Figure 3 Correlation of BrdU incorporation and Ref-1 immunoreactivity. The adjacent sections $\mathbf{A}, \mathbf{B}$ were taken from the far periphery of a section from the retina of a P6 rat previously injected with BrdU to label all proliferating cells. (A, C) BrdU immunohistochemistry; (B, D) Staining for Ref-1. (A, B) Notice the least intense labeling for Ref-1 in the far periphery (arrows) where there is the most BrdU staining, and increased Ref-1 content in the area with less BrdU incorporation (arrowhead). (C, D) Higher magnification of the periphery of the sections shown in A, B. Notice the border between the intense Ref-1 staining in the inner nuclear layer (in D) and the weakly stained neuroblastic layer. Arrows in C point to BrdU labeled cells that delimit the NBL. Arrow in D points to a horizontal cell, while arrowheads indicate outer cells strongly stained for Ref- 1 (see text). Abbreviations as in Figure 1. Calibration bar $=30 \mu \mathrm{m}$ 
band relative to those of a single band of Erk2 detected at approximately $42 \mathrm{kDa}$ were higher in lanes corresponding to older retinae. These results support the specificity of the Ref-1 antibody, and indicate that Ref-1 content increases with retinal development.

\section{Expression of Ref-1 in the brain decreases with development}

We studied the distribution of Ref-1 immunoreactivity in other areas of the central nervous system. In contrast with the retina, we found that immunoreactivity for Ref- 1 in the brain generally decreased with development.

In the hippocampal formation of newborn rats, immunoreactivity for Ref- 1 was the strongest in the dentate gyrus, and slightly less in the CA1-3 fields. Strong and homogeneous immunoreactivity for Ref-1 was found in the nucleus of all labeled cells. In older animals staining became punctate within the nucleus, and decreased to lower levels than in newborn rats (Figure 5). Progressive decrease of Ref-1 immunoreactivity with development was also detected in the cerebellum (not shown).

\section{Ref-1 immunoreactivity is lost in experimentally-induced apoptosis}

In retinal tissue fixed immediately after explantation all nuclei in the ganglion cell layer were stained for Ref-1. At increasing intervals following axotomy of the ganglion cells, there was both an increase in the frequency of cells with condensed chromatin and a decrease in the frequency of Ref-1 positive cells (Figure 6A,B,F). Double labeling with DAPI and the Ref-1 antibody showed that all cells with condensed chromatin were devoid of Ref- 1 staining in the nucleus. However, up to $20 \%$ of the cells with condensed nuclei and a few apparently normallooking cells showed perinuclear staining (Figure 6E,F). In addition, approximately $2 \%$ of the ganglion cells counted in the explants from newborn rat retinae consistently displayed normal-looking chromatin but no Ref-1 staining at 3 or more hours of incubation following axotomy (Figure 6C,D). No ISNEL-positive cells were found at $3 \mathrm{~h}$ after axotomy. Since all cells in the ganglion cell layer of newborn rats are ganglion cells proper (as opposed to displaced amacrine cells), as shown by retrograde labeling with horseradish peroxidase, ${ }^{25}$ and all axotomized ganglion cells degenerate within 2-3 days in explants, ${ }^{26}$ the latter result indicates that the loss of Ref-1 immunoreactivity in the nucleus precedes both the DNA cleavage and chromatin condensation typical of apoptosis.

These results were confirmed with the ISNEL technique to detect DNA fragmentation in situ simultaneously with immunocytochemistry for Ref-1. All apoptotic cells in the GCL were negative for Ref-1, while none of the Ref-1 positive cells were stained with the ISNEL technique (not shown).

As previously shown, ${ }^{3}$ in explants from newborn rats treated with anisomycin for $24 \mathrm{~h}$, inhibition of protein synthesis induced apoptosis in the neuroblastic layer, a region of weak expression of Ref-1. The apoptotic cells again had no Ref-1 staining. In contrast, the ganglion cells, amacrine and horizontal cells, that resist apoptosis induced by anisomycin maintained their high content of Ref-1. The loss of Ref-1 immunoreactivity was also observed in
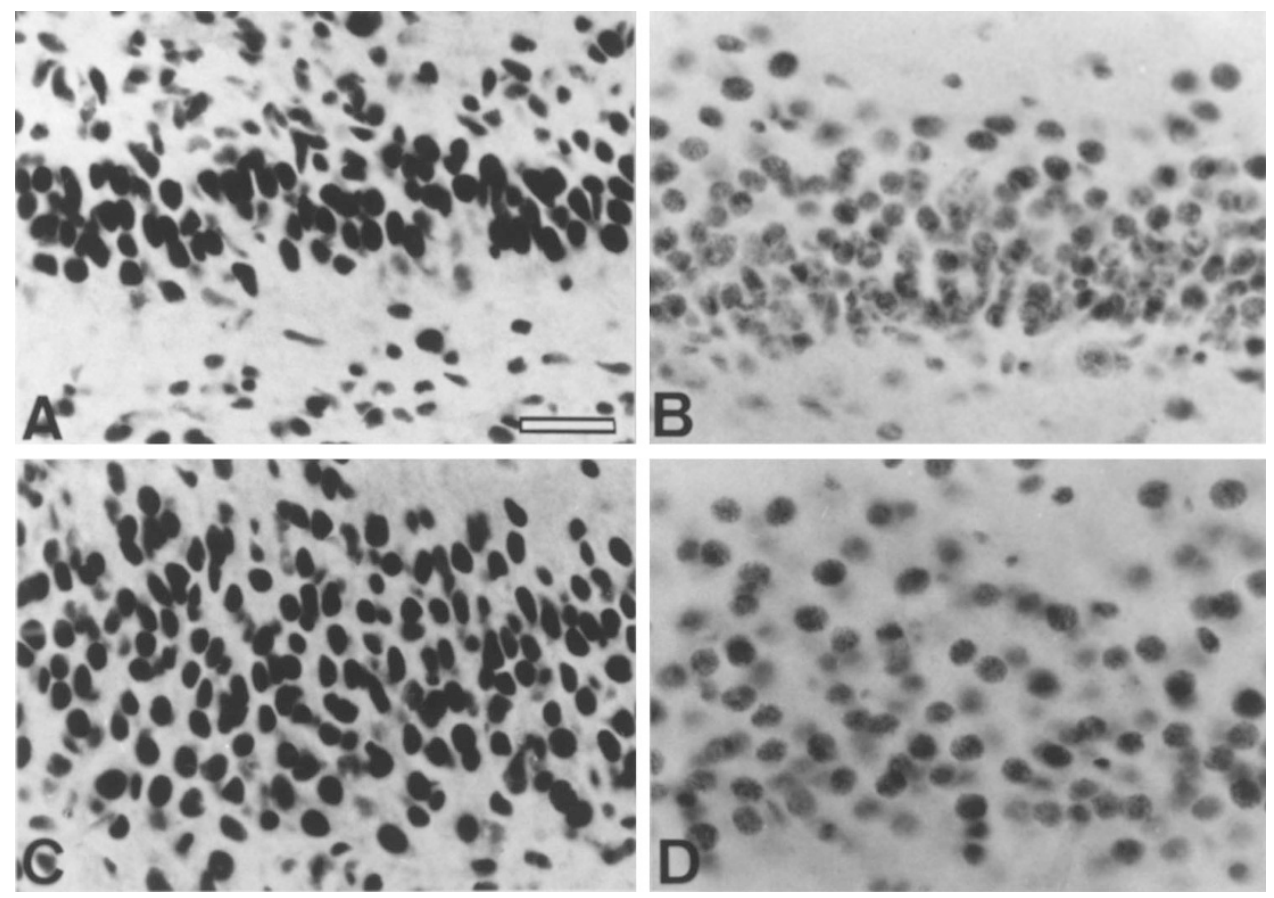

Figure 5 Immunohistochemical detection of Ref-1 in the hippocampal formation of developing rats. (A, B) Dentate gyrus; (C, D) CA1 field. A, C are P0; B, D are P13. Notice the decrease of Ref-1 immunoreactivity with age. Calibration bar $=20 \mu \mathrm{m}$ 

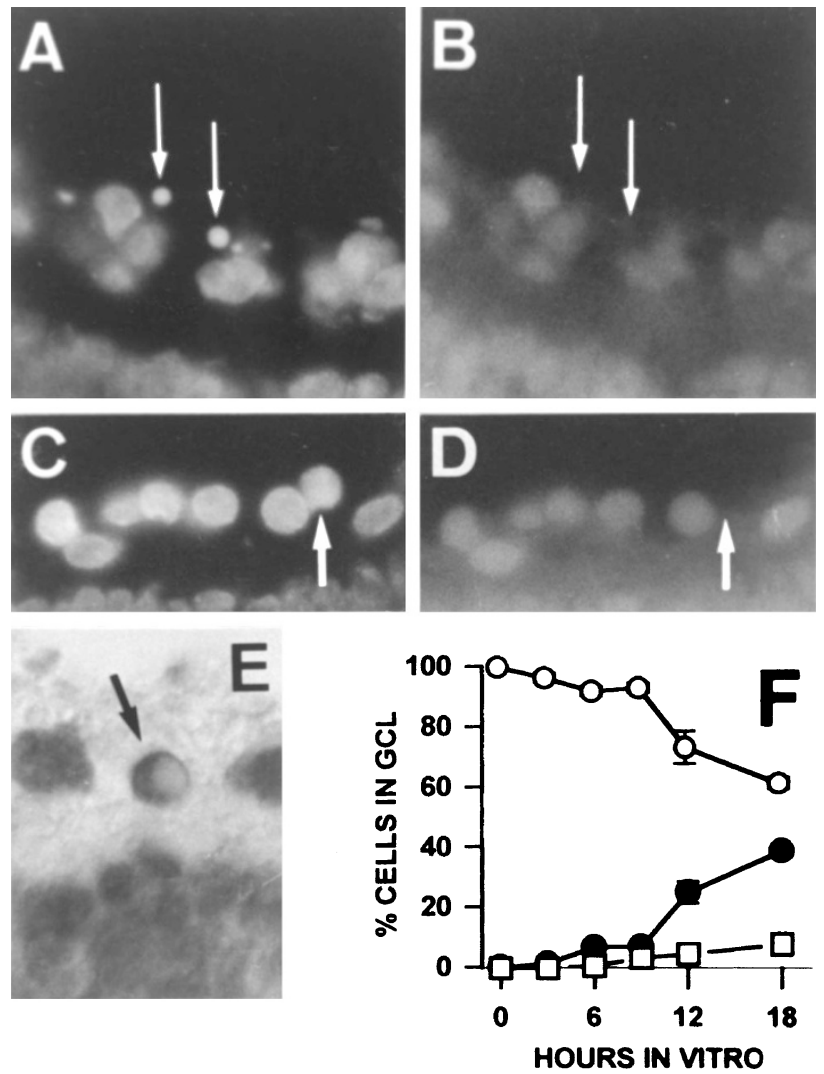

Figure 6 Ref-1 immunoreactivity in the ganglion cell layer of retinal explants (A, C) DAPI staining; (B, D) Ref-1 immunohistochemistry. Notice the lack of Ref- 1 in the condensed nuclei of apoptotic cells (arrows in A, B), and in an uncondensed nucleus (arrows in C, D). (E) Apoptotic body with Ref-1 immunoreactivity restricted to the cytoplasm (HRP-conjugated secondary antibody). (F) Frequencies of cells with nuclear staining for Ref-1 (open circles), apoptotic cells with no Ref-1 immunoreactivity (filled circles), and apoptotic cells with cytoplasmic Ref-1 immunoreactivity (squares), in the ganglion cell layer of $\mathrm{P} 1$ retinal explants at various intervals following explantation (data from simultaneous DAPI and Ref-1 staining illustrated in A-D)

apoptotic cells of the neuroblastic layer following treatment of explants from newborn rat retinae with either 2aminopurine or etoposide, and following gamma irradiation or heat shock (not shown). In contrast, immunoreactivity to the proliferative cell nuclear antigen is maintained in apoptotic nuclei following treatment with either anisomycin (Figure 9 in reference 2) or various other drugs (data not shown).

In retinal explants from older animals, we found that at least two distinct treatments that are likely to affect nonselectively all retinal cells induced apoptosis preferentially in the photoreceptors, that show the least content of Ref-1 among differentiated cells. Thus, the treatment of retinal explants from P6 rats with either thapsigargin, an inhibitor of the endoplasmic reticulum $\mathrm{Ca}^{2+}$-ATPase, or with okadaic acid, a phosphatase inhibitor, induced apoptosis mainly in the outer nuclear layer (Figure 7). The apoptotic cells were devoid of Ref-1 staining, while staining for rhodopsin was maintained.
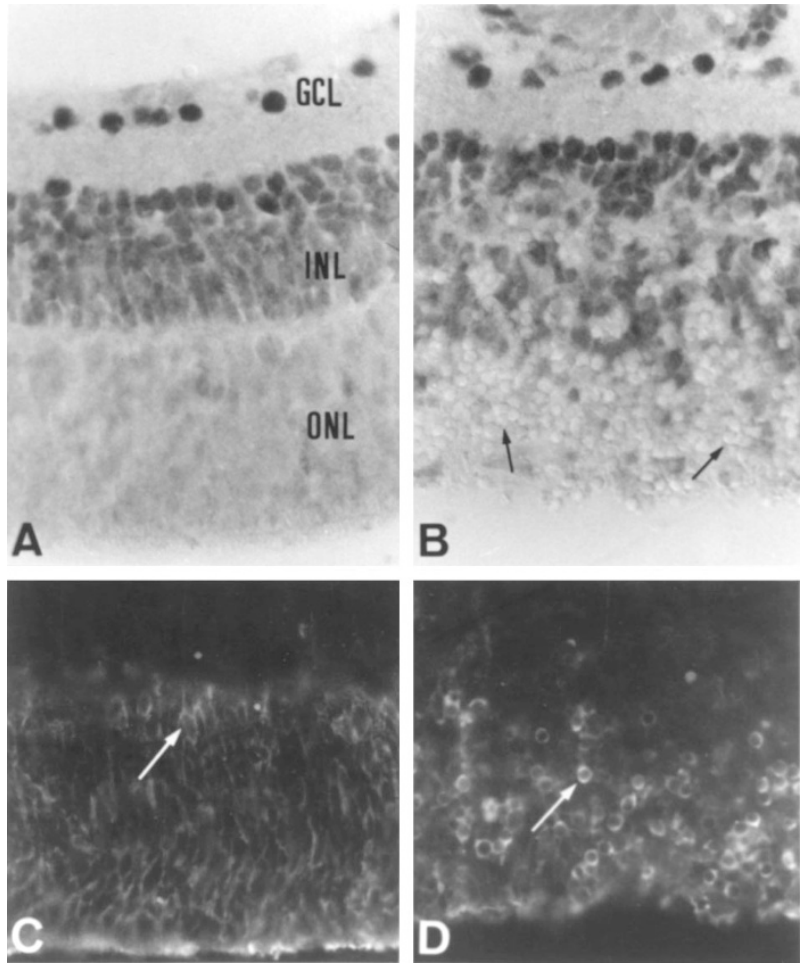

B

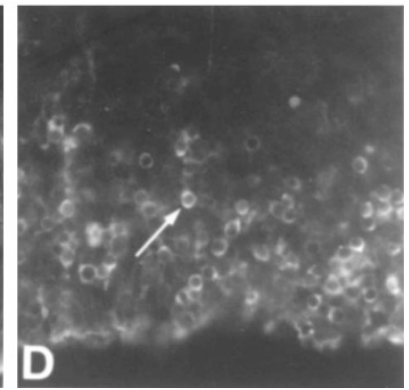

Figure 7 Selective degeneration and loss of Ref-1 immunoreactivity in photoreceptors. (A) Ref-1 staining of a control explant; (B) Ref-1 in explant treated with okadaic acid. Notice the abundant apoptotic bodies devoid of Ref1 staining, located predominantly in the outer nuclear layer. (C) Rhodopsin staining in the outer nuclear layer; (D) Maintenance of rhodopsin staining in the shrunken apoptotic photoreceptors

\section{Prevention of apoptosis is associated with retention of Ref-1 immunoreactivity}

When the apoptosis of ganglion cells was blocked with anisomycin, the decrease of Ref-1 immunoreactivity was prevented (Figure $8 \mathrm{~A}-\mathrm{C}$ ). A similar result was obtained following treatment of the explants with 2-aminopurine, that also prevents the death of the axotomized ganglion cells. ${ }^{27}$

Inhibition of protein synthesis also blocked apoptosis of photoreceptors induced by either thapsigargin or okadaic acid (Figure 8D,E), and in these conditions the immunoreactivity for Ref-1 in the photoreceptors was equivalent to that found in the outer nuclear layer of explants maintained in control conditions (c.f. Figure 7A).

\section{Loss of Ref-1 immunoreactivity is associated with naturally occurring neuron death in vivo}

Naturally occurring cell death was examined both in the retina and in the brain either at birth or at 7 days after birth. In the retina of newborn rats apoptotic cells detected by condensed chromatin lacked immunoreactivity for Ref-1, while viable cells in the GCL were strongly stained (Figure 9A,B). Occasional viable cells were negative for Ref-1, which may correspond to early stages of programmed cell death. In addition, in both 

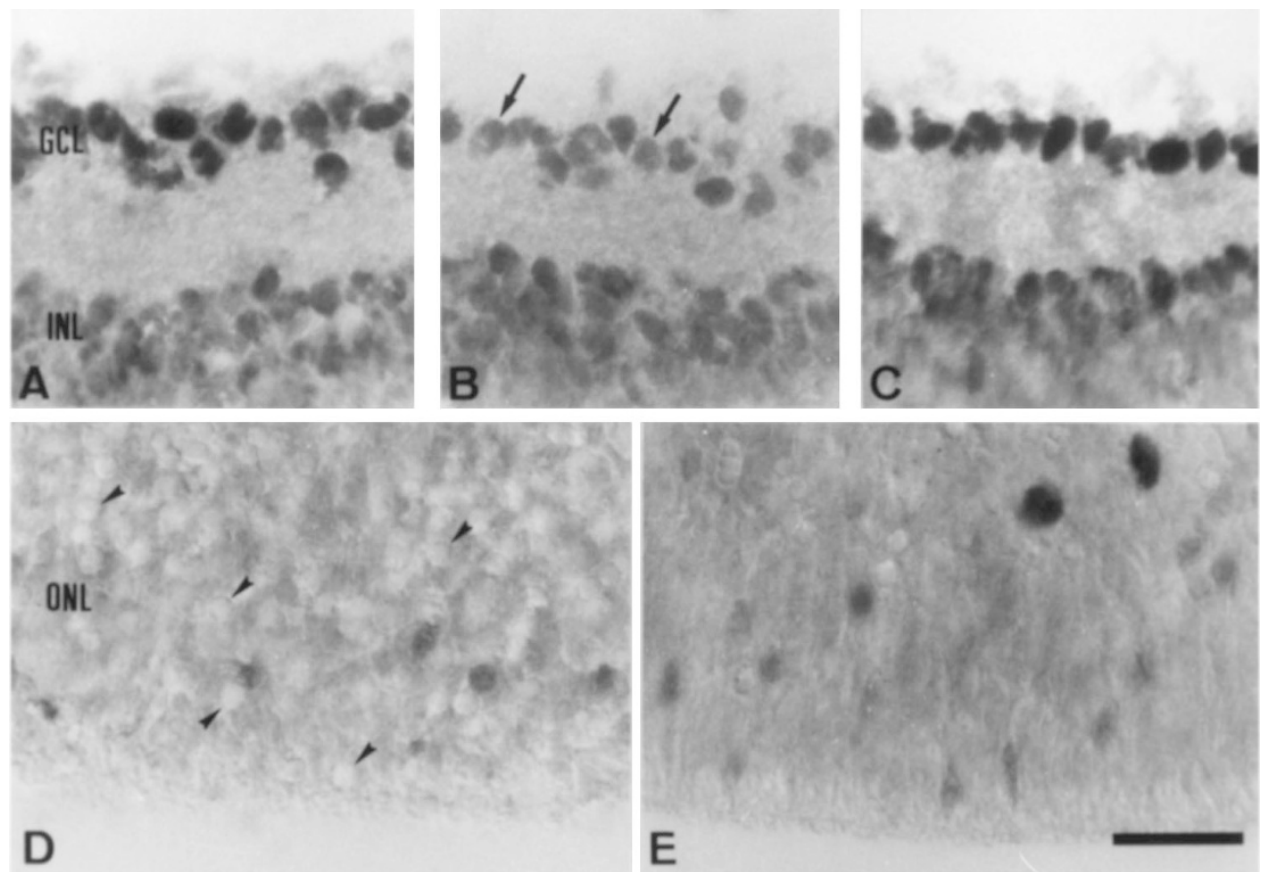

Figure 8 Blockade of Ref-1 degradation with anisomycin. (A-C) Ganglion cell layer of P7 retinal explants immediately after explantation (A), and after 1 day in vitro in either control medium (B) or with $1 \mu \mathrm{g} / \mathrm{ml}$ anisomycin (C). Notice that Ref-1 immunoreactivity in axotomized ganglion cells (arrows) is decreased to a level similar to the amacrine cells of the inner nuclear layer in the same section, while anisomycin preserves the more intense staining of the former. The results are representative of 1-4 rounds of immunohistochemistry in each of eight distinct experiments. (D, E) Outer nuclear layer of P5 explants incubated for 1 day with thapsigargin $10 \mathrm{nM}$ either without (D) or together with anisomycin $1 \mu \mathrm{g} / \mathrm{ml}(\mathbf{E})$. Notice the lack of Ref-1 immunoreactivity in apoptotic photoreceptors (arrowheads in D), and preservation of immunoreactivity upon treatment with anisomycin (compare with Figure 7A). Calibration bar=30 $\mu \mathrm{m}$
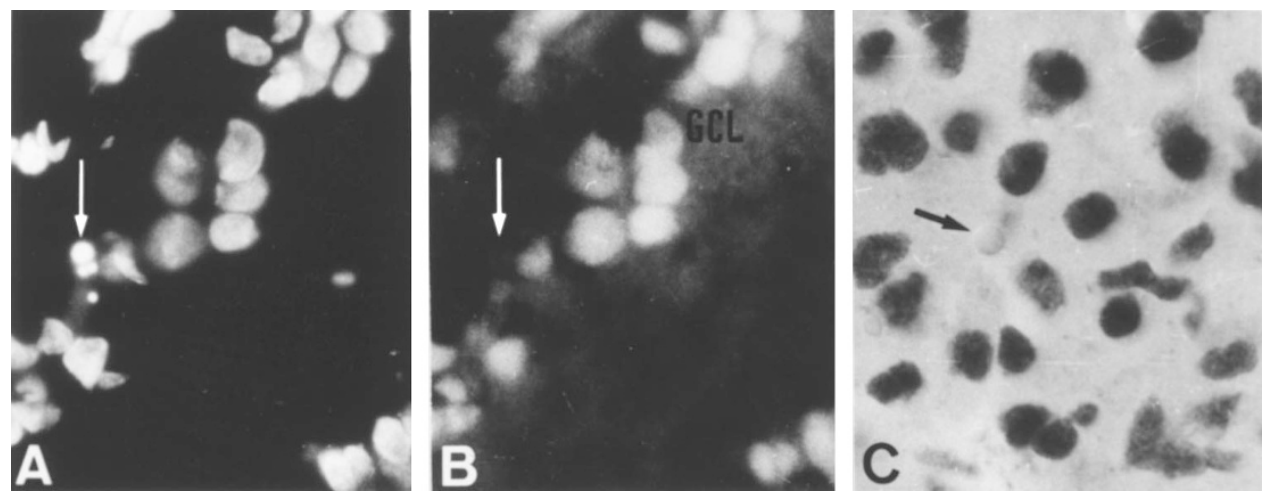

Figure 9 Loss of Ref-1 in apoptotic cells in vivo. (A, B) Bisbenzimide staining (A) and Ref-1 immunocytochemistry (B) of the ganglion cell layer of the retina of a newborn rat. Notice the Ref-1 negative clumps of condensed chromatin from an apoptotic cell (arrow), contrasting with the Ref-1 staining of all healthy nuclei. (C) Section from the hippocampus of a newborn rat, stained with the antibody for Ref-1. Notice the Ref-1 negative apoptotic body (arrow)

the hippocampus and the cerebellum we found naturally occurring apoptotic cells negative for Ref- 1 surrounded by healthy Ref-1 immunoreactive cells. (Figure 9C).

\section{Discussion}

Our results show that Ref- 1 is associated with differentiation of retinal cells. The Ref- 1 content increases during postnatal development in the retina, while the levels of the same protein in the brain decrease during the same period in developing rats. The proliferative zone in the retina contained little Ref- 1 , and differentiated cells such as ganglion cells, earlydeveloping amacrines and horizontal cells showed high amounts of the protein even at birth. Photoreceptors contained less Ref-1 than the other retinal neurons, but higher amounts than in the proliferative zone. The immunoreactivity for Ref-1 was maintained high at least until P45. Ref1 was found in the nucleus of all retinal cells, except for a few naturally occurring degenerating profiles which were negative.

Previous work has shown evidence for regulated expression of Ref-1 during neural development. High 
levels of Ref- 1 mRNA were described in the proliferative zone of various brain regions, followed by a steady decrease associated with development. ${ }^{28}$ An exception to this pattern was the maintained expression reported in the hippocampal formation. The pyramidal and granule cell layers expressed Ref- 1 at the highest level among most other brain regions at postnatal day $21 .^{28}$ Our data, however, indicate that even within the hippocampal formation the amount of Ref-1 protein tends to decrease during postnatal development. Previously reported differences between the steady-state levels of Ref- 1 in distinct brain areas, such as the higher level in the dentate gyrus than in the CA1 field of the hippocampal formation ${ }^{24}$ were also found in our material.

Immunoreactivity for Ref-1 was reported in the hippocampus, cerebellum and hypoglossal nucleus of the brainstem of a 70 year-old human brain. ${ }^{29}$ The subcellular location of the protein was described as cytoplasmic in neurons of the dentate gyrus and region CA4, and both cytoplasmic and nuclear in cerebellar Purkinje cells. Ref-1 was also detected in the cytoplasmic Niss/ substance in the hypoglossal nucleus of the brainstem. Distinct from those findings, we found that in the rat the subcellular location of Ref-1 was nuclear in normal cells of the hippocampus, cerebellum and retina at all ages. In contrast, some apoptotic cells showed perinuclear Ref-1 immunoreactivity.

The progressive increase in the expression of Ref-1 protein in the developing retina disclosed in the present work differs, therefore, from the pattern in the brain. It is, however, similar to that in the testis, in which Ref-1 mRNA was found to increase after withdrawal from the cell cycle. Higher levels of Ref-1 mRNA expression were detected over round spermatids, which are post-meiotic cells, than in spermatogonia or spermatocytes. ${ }^{23}$

Our results suggest that Ref-1 may be an anti-apoptotic protein. Evidence for this hypothesis stems both from the loss of Ref-1 immunoreactivity in apoptotic nuclei, and for the finding of a consistent increase in the frequency of apparently healthy cells devoid of Ref- 1 staining within the nucleus following an apoptotic insult and preceding their demise. In addition, the photoreceptors that express relatively low levels of Ref- 1 were the most sensitive to the induction of apoptosis by both thapsigargin and okadaic acid, and blockade of apoptosis in various circumstances was accompanied by the preservation of nuclear Ref-1. These observations are in line both with previous reports of increased sensitivity to DNA damage following depletion of APE/Ref-1 in cell lines, ${ }^{18,19}$ and with decreased Ref-1 levels preceding apoptosis-associated DNA fragmentation after an ischemic insult. ${ }^{22}$ In addition, preliminary results show that sympathetic neurons in culture lose Ref-1 immunoreactivity upon NGF withdrawal, and addition of NGF prevents both their death and the loss of Ref-1 (LB Chiarini and AM Tolkovsky, unpublished results).

This study shows for the first time a possible link between the course of neural differentiation and an antiapoptotic role of Ref-1. Inhibition of protein synthesis blocks apoptosis both of axotomized ganglion cells ${ }^{3}$ and of relatively differentiated photoreceptors treated with either thapsigargin or okadaic acid, whereas it induces apoptosis of undifferentiated postmitotic cells. ${ }^{2}$ This suggests that protein synthesis is critical for survival early after withdrawal from the cell cycle. The period between the last mitosis and the achievement of a fully differentiated state is characterized by an intense synthesis of Ref-1, which may explain the sensitivity of early postmitotic cells to the inhibition of protein synthesis. On the other hand, prevention by anisomycin of apoptosis in either the differentiated ganglion cells or photoreceptors was accompanied by retention of Ref-1, suggesting that the apoptotic insults induce degradation of Ref-1 along the $24 \mathrm{~h}$ in vitro, and that inhibition of protein synthesis blocks this degradation.

Notwithstanding, proliferating cells are also resistant to inhibition of protein synthesis. This suggests that Ref- 1 may have an important anti-apoptotic function in retinal cells only following their withdrawal from the cell cycle. Proliferating cells may activate various mechanisms of recognition and repair of DNA damage related to the cell cycle. ${ }^{30,31}$ In contrast, the repair endonuclease APE/Ref-1 may be particularly important for differentiated nerve cells, because AP sites are the most common form of DNA damage under physiological conditions, ${ }^{32}$ and the mature retinal tissue has not been shown to maintain stem cells that might provide repair at the level of the tissue by cell replacement. $^{32}$ Therefore, increased levels of Ref-1 in postmitotic retinal cells may be of advantage to confer resistance to programmed cell death triggered by DNA damage. This idea is apparently difficult to reconcile with the decreasing levels of Ref- 1 in the brain. However, the retina is constantly exposed to electromagnetic radiation, including UV-A, which may conceivably lead to a higher risk of DNA damage leading to a higher demand for DNA repair mechanisms than in the brain.

It has also been demonstrated that astrocytes are more resistant than neurons to programmed cell death triggered by X-irradiation, and that DNA repair is more efficient in astrocytes than in neurons. ${ }^{33}$ In addition, astrocytes express higher amounts of Ref- 1 than neurons in the brain. ${ }^{24}$ These data together are consistent with an antiapoptotic role for Ref- 1 through its DNA repair activity.

On the other hand, the fact that inhibition of protein synthesis blocked apoptosis induced by the $\mathrm{Ca}^{2+}$-ATPase inhibitor thapsigargin, suggests a calcium-induced activation of pro-apoptotic genes. Recent evidence indicates that Ref- 1 is directly involved in calcium-induced gene repression, ${ }^{34}$ which is consistent with the hypothesis that the selective sensitivity of photoreceptors to apoptosis induced by thapsigargin may be related to their low Ref- 1 content. This alternative function of Ref-1 may be also relevant to retinal development because of the increasing expression of both calcium-binding proteins, such as calretinin and calbindin, and neurotransmitter receptors that mobilize extracellular calcium, ${ }^{35-37}$ which suggest a progressive increase of calcium-mediated processes in differentiated retinal cells.

Ref- 1 is also a regulator of various transcription factors that may be important during differentiation of the retina. For example, ATF2, the activation of which is regulated by 
Ref- $1,{ }^{11}$ shows a developmental pattern of expression somewhat similar to Ref- 1 in the retina of postnatal rats (unpublished results). A previous report described both Ref1 decrease and c-Jun increase the hippocampus after an ischemic insult, but suggested that the two events were not related. ${ }^{22}$ In the retina, we found c-Jun immunoreactivity associated with apoptosis in the developing retina, ${ }^{38}$ similar to the axotomized retinal ganglion cells in adult rats. ${ }^{39}$ However, the location of c-Jun immunoreactivity in apoptotic cells was predominantly cytoplasmic, suggesting that this transcription factor may be inactive. A link between Ref-1 and translocation of c-Jun is possible because the activity of $c$-Jun is modulated by Ref- 1 through reduction of a cystein within the DNA binding domain, ${ }^{11,40}$ and this residue lies immediately adjacent to the nuclear localization signal (NLS). This cystein was not directly shown to control translocation. However, there is evidence that in v-Jun, where serine replaces the relevant cystein, the phosphorylation of this serine controls the translocation of v-Jun. ${ }^{41}$ If indeed reduction of the cystein adjacent to the NLS should control the translocation of c-Jun, then the accumulation of c-Jun in the cytoplasm of apoptotic cells may be a consequence of the loss of Ref- 1 .

In conclusion, our data show that the expression of Ref1 is regulated both during differentiation and during apoptosis of retinal neurons, and suggest that Ref-1 may play an anti-apoptotic role in the central nervous system related with progressive cell differentiation.

\section{Materials and Methods}

\section{Preparation of retinae and brains}

Lister hooded rats of various ages were deeply anesthetized by inhalation of chloroform and perfused through the heart with saline followed by either $4 \%$ paraformaldehyde in phosphate buffer $\mathrm{pH} 7.2$, or sequentially perfused with $4 \%$ paraformaldehyde in acetate buffer $\mathrm{pH} 6.5$ followed by the same fixative in borate buffer $\mathrm{pH} 11 .{ }^{42}$ Both protocols produced similar results, but the quality of both fixation and immunohistochemistry was the best with the sequential procedure at variable $\mathrm{pH}$. The eyecups containing the retina in situ were oriented to allow sagital sectioning, and cut frozen at $10 \mu \mathrm{m}$ thickness, after removal of the cornea and lens. The brains of the perfused rats were postfixed with $4 \%$ paraformaldehyde for 1 day and then soaked in $20 \%$ sucrose. Frozen sagital sections of $10 \mu \mathrm{m}$ thickness were cut in a cryostat and mounted on gelatinized slides. The slides were kept at $4^{\circ} \mathrm{C}$ until processed.

\section{Detection of proliferating cells}

The nucleotide analog bromodeoxiuridine (BrdU) was injected in 5 day-old rats. Two injections of $60 \mathrm{mg} / \mathrm{kg} \mathrm{b} . \mathrm{w}$. were made at an interval of $23 \mathrm{~h}$ and $1 \mathrm{~h}$ after the second injection the rats were perfused as described above. The incorporated BrdU was detected by immunohistochemistry following an acid wash, ${ }^{43}$ using a monoclonal antibody (RPN 202, Amersham), and developed with an anti-mouse ABC kit (Vector) using di-aminobenzidine as chromogen.

\section{Cultures of retinal explants}

The rat pups were killed either by decapitation or by inhalation of chloroform, and their eyes were rapidly removed. The retinae were dissected out of the eyeballs and cut into small pieces $\left(1 \mathrm{~mm}^{2}\right)$ in culture medium (Basal Medium of Eagle with $5 \%$ fetal calf serum). The tissue fragments were incubated at $37^{\circ} \mathrm{C}$ in an atmosphere of $5 \% \mathrm{CO}_{2}$ and $95 \%$ air, in an orbital shaker at $80-90$ r.p.m. for the required periods of time. The tissue was fixed by immersion in $4 \%$ paraformaldehyde in phosphate buffer $\mathrm{pH} 7.2$ for at least $40 \mathrm{~min}$, followed by $20 \%$ sucrose in the same buffer. The explants were oriented under a dissecting microscope in an aluminum chamber with OCT embedding medium, and $10 \mu \mathrm{m}$ thick transverse sections were cut in a cryostat.

\section{Treatments}

The explants were maintained in vitro for various periods of time. Drugs were added at the beginning of the incubation period. Treatments used to modulate apoptosis included incubations with the inhibitor of the endoplasmic reticulum $\mathrm{Ca}^{2+}$-ATPase thapsigargin $(10 \mathrm{nM})$, the phosphatase inhibitor okadaic acid $(10 \mathrm{nM})$, the inhibitor of protein synthesis anisomycin $(1 \mu \mathrm{g} / \mathrm{ml})$, the non-specific protein kinase inhibitor 2-aminopurine (10 $\mathrm{mM})$, the topoisomerase II inhibitor etoposide $(3 \mu \mathrm{M})$, gamma irradiation (2 Gy) and heat stress $\left(42^{\circ} \mathrm{C}\right.$ for $1 \mathrm{~h})$.

\section{Detection of apoptotic cells}

Apoptosis was detected either by the condensation of chromatin stained with neutral red or with the DNA-intercalating dyes 4'-6-diamino-2-phenylindole (DAPI) or bisbenzimide (Hoechst 33258), or with in situ DNA nick end labeling (ISNEL).

For ISNEL, the sections were incubated with $0.5 \%$ Triton X-100 for $15 \mathrm{~min}$. The slides were washed with PBS pH 7.4 and pre-incubated in terminal-deoxyribonucleotidyl-transferase (TdT) buffer for $20 \mathrm{~min}$. After that the sections were incubated overnight at $37^{\circ} \mathrm{C}$, in a solution containing $0.5 \mathrm{U} / \mu \mathrm{l}$ TdT (Gibco BRL), $0.2 \mathrm{nmol} / \mu \mathrm{l} \mathrm{dATP}$ and $0.013 \mathrm{nmol} / \mu$ l biotin-14-dATP inTdT buffer. The reaction was stopped with $20 \mathrm{mM}$ EDTA for $5 \mathrm{~min}$ and the slides were washed with PBS. Permanent staining was obtained with HRP-conjugated streptavidin (reagents $A+B$ ) from an $A B C$ Kit (Vector), followed by diaminobenzidine according to the manufacturer's directions. For fluorescent staining we used streptavidin conjugated with FITC (Amersham).

\section{Immunohistochemistry}

Tissue sections were incubated with $0.5 \%$ Triton X-100 for $15 \mathrm{~min}$ and washed with PBS, followed by incubation with either normal serum or a blocking albumin solution for $30 \mathrm{~min}$. Then, the sections were reacted with rabbit polyclonal antibodies raised against residues $299-318$ of the Ref-1 protein (sc-334, Santa Cruz Biotechnology). Incubations with the primary antibody were carried out at $37^{\circ} \mathrm{C}$, either overnight at $1: 1000$ or at $1: 100$ for $3 \mathrm{~h}$. Permanent staining was obtained with an HRP-ABC kit (Vector) using di-aminobenzidine as chromogen. Fluorescent staining was done with an anti-rabbit IgG secondary antibody conjugated with Texas Red (Amersham). Immunocytochemical procedures in the absence of the primary antibody produced no staining of the retinal tissue.

Additional procedures employed in some experiments were the immunohistochemistry for the cell differentiation markers calretinin using a polyclonal antibody (Chemicon), calbindin using a monoclonal 
antibody (Sigma) and rhodopsin labeled with monoclonal antibody rho 4D2 (kindly provided by Dr. R Molday), respectively. The monoclonal antibodies were developed with an anti-mouse $\lg G$ secondary antibody conjugated with FITC (Amersham).

\section{Western blots}

Fractions of $40 \mu \mathrm{g}$ of total protein extracts from various samples of retinal tissue were resolved in $12.5 \%$ SDS-PAGE, and electrotransferred $(120 \mathrm{~mA})$ onto a nitrocellulose filter for $2 \mathrm{~h}$ at $4{ }^{\circ} \mathrm{C}$. Transfer was verified by staining the membrane with Ponceau Red. Following the transfer the membrane was incubated at room temperature in blocking buffer (TBS+0.2\% Tween $+5 \%$ skimmed milk). Incubation with the Ref- 1 antibody was carried out for $7 \mathrm{~h}$ at room temperature. Following three washes with $0.05 \%$ Tween-20 in TBS, the membrane was incubated with HRP-conjugated anti-rabbit $\operatorname{lgG}$ at room temperature for $2 \mathrm{~h}$. Unbound antibody was washed out and the membrane was developed with an ECL kit (Amersham) according to the manufacturer's directions. Then, the membrane was stripped according to the ECL instructions and re-blotted with a polyclona antibody to Erk2 (Santa Cruz Biotechnology) for $2 \mathrm{~h}$ at room temperature, as a loading control protein. Subsequent processing was carried out as described above.

\section{Acknowledgements}

This investigation was supported by grants and fellowships from CNPq, CAPES, FINEP, FAPERJ, FUJB-UFRJ and PRONEX-MCT. We thank José Nilson dos Santos, Levi R de Amorim, Wilson R de Lima and Jose $F$ Tiburcio for technical support, and Aviva M Tolkovsky and Stevens K Rehen for critical reading of the manuscript.

\section{References}

1. Cowan WM (1981) The development of the vertebrate nervous system: An overview. In: Development in the Nervous System. Garrod DR and Feldman JD, (eds) Cambridge, Cambridge University Press pp. 3-35

2. Linden R, Rehen SK and Chiarini LB (1999) Apoptosis in developing retinal tissue. Progr. Retinal Eye Res. 18: 133-165

3. Rehen SK, Varella MH, Freitas FG, Moraes MO and Linden R(1996) Contrasting effects of protein synthesis inhibition and of cyclic AMP on apoptosis in the developing retina. Development 122: 1439-1448

4. Dowling JE (1987) The Retina. The Belknap Press of Harvard University, Cambridge, Mass

5. Oppenheim RW (1991) Cell death during development of the nervous system. Ann. Rev. Neurosci. 14: 453-501

6. Beazley LD, Perry VH, BakerB and Darby JE (1987) An investigation into the role of ganglion cells in the regulation of division and death of other retinal cells. Dev. Brain Res. 33: 169-184

7. Barnstable CJ (1987) A molecular view of vertebrate retinal development. Mol. Neurobiol. 1: 9-46

8. Von Bartheld CS (1998) Neurotrophins in the developing and regenerating visual system. Histol. Histopathol. 13: 437-459

9. Robson CN and Hickson ID (1991) Isolation of cDNA clones encoding a human apurinic/apyrimidinic endonuclease that corrects DNA repair and mutagenesis defects in E. coli xth (endonuclease III) mutants. Nucleic Acids Res. 19: 5519 5523

10. Demple B, Herman T and Chen DS (1991) Cloning and expression of APE, the cDNA encoding the major human apurinic endonuclease: Definition of a family of DNA repair enzymes. Proc. Natl. Acad. Sci. USA 88: 11450-11454

11. Xanthoudakis S and Curran $T$ (1992) Identification and characterization of Ref-1, a nuclear protein that facilitates AP-1 DNA-binding activity. EMBO J. 11: 653 665
12. Xanthoudakis S, Miao G and Curran T (1994) The redox and DNA-repair activities of Ref-1 are encoded by nonoverlapping domains. Proc. Natl. Acad. Sci. USA 91: 23-27

13. Xanthoudakis S, Smeyne RJ, Wallace JD and Curran T (1996) The redox/DNA repair protein Ref- 1 is essential for early embryonic development in mice. Proc. Natl. Acad. Sci. USA 93: 8919-8923

14. Jayaraman L, Murthy KGK, Zhu C, Curran T, Xanthoudakis SandPrives C (1997) Identification of redox/repair protein Ref-1 as a potent activator of p53. Genes Dev. 11: 558-570

15. Xanthoudakis S, Miao G, Wang F, Pan YCE and Curran T (1992) Redox activation of Fos-Jun DNA binding activity is mediated by a DNA repair enzyme. EMBO J. 11: 3323-3335

16. Mumane JP (1995) Cell cycle regulation in response to DNA damage in mammalian cells: A historical perspective. Cancer Metastasis Rev. 14: 17-29

17. Karin M, Liu Zg and Zandi E (1997) AP-1 function and regulation. Curr. Opin. Cell Biol. 9: 240-246

18. Walker LJ, Craig RB, Harris AL and Hickson ID (1994) A role for the human DNA repair enzyme HAP1 in cellular protection against DNA damaging agents and hypoxic stress. Nuclei Acids Res. 22: 4884-4889

19. Ono Y, Furuta T, Ohmoto T, Akiyama Kand Seki S(1994) Stable expression in rat glioma cells of sense and antisense nucleic acids to a human multifunctionalDNA repair enzyme, APEX nuclease. Mutation Res. 315: 55-63

20. Robertson KA, Hill DP, XuY, Liu L, Van Epps S, Hockenbery DM, ParkJR, Wilson TM and Kelley MR (1997) Down-regulation of apurinic/apyrimidinic endonuclease expression is associated with the induction of apoptosis in differentiating myeloid leukemia cells. Cell Growth Differ. 8: 443-449

21. Yao KS, Clayton Mand O'DwyerPJ (1995) Apoptosis in human adenocarcinoma HT29 cells induced by exposure to hypoxia. J. Natl. Cancer Inst. 87: 117-122

22. Walton $M$, Lawlor $P$, Sirimanne $E$, Williams $C$, Gluckman $P$, Dragunow $M$ and Takahashi R (1997) Loss of Ref-1 protein expression precedes DNA fragmentation in apoptotic neurons. Brain Res. Mol. Brain Res. 24: 1381-1385

23. Wilson TM, Rivkees SA, Deutsch WA and Kelley MR (1996) Differential expression of the apurinic/apyrimidinic endonuclease (APE/ref-1) multifunctional DNA base excision repair gene during fetal development and in the adult rat brain and testis. Mutation Res. 362: $237-248$

24. Dragunow M (1995) Ref-1 expression in adult mammalian neurons and astrocytes. Neurosci. Lett. 191: 189-192

25. Perry VH, Henderson Z and Linden R (1983) Postnatal changes in retinal ganglion cell and optic axon populations in the pigmented rat. J. Comp. Neurol. 219: $356-368$

26. Rehen SK, Ary-Pires R and Linden R (1993) Intraretinal neurotrophic activity prevents the degeneration of ganglion cells in retinal explants. Braz. J. Med. Biol. Res. 26: 955-959

27. Varella MH, Correa DF, Campos CBL, Chiarini LB and Linden R (1997) Protein kinases selectively modulate apoptosis in the developing retina in vitro. Neurochem. Int. 31: 217-227

28. Ono Y, Watanabe M, Inoue Y, Ohmoto T, Akiyama K, Tsutsu Kand Seki S (1995) Developmental expression of APEX nuclease, a multifunctional DNA repair enzyme, in mouse brains. Dev. Brain. Res. 86: 1-5

29. Duguid JR, Eble JN, Wilson TM and Kelley MR (1995) Differential cellular and subcellular expression of the human multifunctional apurinic/apyrimidinic endonuclease (APE/ref-1) DNA repair enzyme. Cancer Res. 55: 6097-6102

30. Smith ML and Fomace Jr AJ (1996) Mammalian DNA damage-inducible genes associated with growth arrest and apoptosis. Mutat. Res. 340: 109-124

31. Liu VF, Boubnov NV and Weaver DT (1995) Cell cycle checkpoints and repair of ionizing radiation damage. Stem. Cells 13: 117-128

32. Lindhal T and Nyberg B (1972) Rate of depurination of native deoxyribonucleic acid. Biochem. 11: 3810-3818

33. Gobbel GT, Bellinzona M, Vogt AR, Gupta N, Fike JR and Chan PH (1998) Response of postmitotic neurons to X-irradiation: implications for the role of DNA damage in neuronal apoptosis. J. Neurosci. 18: 147-155

34. Okazaki T, Chung UI, Nishishita T, Ebisu S, Usuda S, Mishiro S, Xanthoudakis S, Igarashi Tand OgataE (1994)A redox factor protein, Ref- 1 is involved in negative gene regulation by extracellular calcium. J. Biol. Chem. 269: 27855-27862

35. Yan XX (1997) Prenatal development of calbindin D-28K and parvalbumin immunoreactivities in the human retina. J. Comp. Neurol. 377: 565-576 
36. Nag TC and Wadhwa S (1996) Calbindin and parvalbumin immunoreactivity in the developing and adult human retina. Brain Res. Dev. Brain Res. 93: 23-32

37. Wong ROL (1995) Effects of glutamate and its analogs on intracellular calcium levels in the developing retina. Visual Neurosci. 12: 907-917

38. Chiarini LB, Freitas FG and Linden R (1997) Association of Ref-1 and c-Jun with apoptosis and differentiation in the developing retina. Soc. Neurosci. Abstr. 23: 898

39. Isenmann S, and Bahr M (1997) Expression of C-Jun protein in degenerating retinal ganglion cells after optic nerve lesion in the rat. Exp. Neurol. 147: 28-36

40. Abate C, Patel L, Rauscher FJ and Curran T (1990) Redox regulation of the Fos and Jun DNA-binding activity in vitro. Science 249: 1157-1161
41. Tagawa T, Kuroki T, Vogt PK and Chida K (1995) The cell cycle-dependent nuclear import of v-Jun is regulated by phosphorylation of a serine adjacent to the nuclear localization signal. J. Cell Biol. 130: 255-263

42. Berod A, Hartman BK and Pujol JF (1981) Importance of fixation in immunohistochemistry: use of formaldehyde solutions at variable $\mathrm{pH}$ for the localization of tyrosine hydroxylase. J. Histochem. Cytochem. 29: 844-850

43. Menezes JR, Smith CM, Nelson KC and Luskin MB (1995) The division of neuronal progenitor cells during migration in the neonatal mammalian forebrain. Mol. Cell. Neurosci. 6: 496-508 